\title{
Quantification of Botrytis cinerea in Grapevine Bunch Trash by Real-Time PCR
}

\author{
Melissa Si Ammour, ${ }^{1}$ Giorgia Fedele, ${ }^{1}$ Caterina Morcia, ${ }^{2}$ Valeria Terzi, ${ }^{2}$ and Vittorio Rossi ${ }^{1, \dagger}$ \\ ${ }^{1}$ Department of Sustainable Crop Production (DI.PRO.VE.S.), Università Cattolica del Sacro Cuore, 29122 Piacenza, Italy \\ ${ }^{2}$ Research Centre for Genomics and Bioinformatics (CREA-GB), Council for Agricultural Research and Economics, 29017 Fiorenzuola \\ d'Arda, Italy \\ Accepted for publication 18 February 2019
}

\begin{abstract}
Quantification of colonization of grape bunch trash by Botrytis cinerea is crucial for Botrytis bunch rot (BBR) control. A previously developed quantitative polymerase chain reaction (qPCR) method was adapted to quantify $B$. cinerea DNA in grape bunch trash, and a colonization coefficient (CC) was calculated as the ratio between the DNA concentrations of $B$. cinerea and of Vitis vinifera. CC values increased linearly with the number of conidia of $B$. cinerea or the quantity of mycelium of $B$. cinerea added to the bunch trash increased. $C C$ values also increased linearly in bunch trash samples containing increasing percentages of $B$. cinerea-colonized bunch trash; in the latter samples, $\mathrm{CC}$ values were correlated with subsequent assessments of $B$. cinerea
\end{abstract}

ABSTRACT colonization of trash (as determined by plating on agar) and sporulation on the trash (as determined by spore counts after incubation in humid chambers). The qPCR assay was also validated using trash collected from bunches treated or not treated with fungicides in three vineyards in two seasons. CC values reflected the reduction in sporulation and in latent infections of mature berries caused by fungicide application. The qPCR assay enables rapid, specific, sensitive, and reliable quantification of the degree of colonization of bunch trash by $B$. cinerea, which makes it a useful tool for studies of the epidemiology and management of BBR.

Keywords: colonization rate, gray mold, sporulation potential
Botrytis bunch rot (BBR) is an economically important disease of grapevines (Vitis vinifera L.) and is caused by the fungus Botrytis cinerea Pers.: Fr (Elmer and Michailides 2007). Control of BBR is challenging for the following reasons: (i) B. cinerea can function as a saprophyte, necrotroph, or parasite; (ii) the fungus can overwinter and sporulate on multiple inoculum sources (including bunch trash, leaf trash, and rotted berries); (iii) grapevines are susceptible at multiple growth stages; (iv) multiple infection pathways exist; and (v) infections can occur under a wide range of environmental conditions, which differ among infection pathways (Ciliberti et al. 2015a, 2015b, 2016; Elad et al. 2007; Elmer and Michailides 2007; Hill et al. 2014; Nair et al. 1995).

After flowering, the pathogen is able to saprophytically colonize the "bunch trash" (i.e., the dead stamens, aborted flowers, aborted berries, calyptras, tendrils, and leaf pieces retained within developing bunches) (Seyb et al. 2000). Under favorable conditions, the fungus produces abundant conidia on the colonized bunch trash, and these conidia are a source of inoculum for berry infection, mainly after veraison. Elmer and Michailides (2007) referred to these phenomena as infection pathways III (conidial infection and extensive colonization of floral debris in grape bunches), IV (conidial accumulation within the developing bunch), and $\mathrm{V}$ (conidial infection of ripening fruit), respectively.

Bunch trash colonized early by $B$. cinerea is a major source of berry infection (Elmer and Michailides 2007) and has been related to the severity of BBR at harvest (Holz et al. 2003; Keller et al. 2003; Nair et al. 1995; Viret et al. 2004). Thus, reducing the quantity of bunch trash, as well as reducing colonization of bunch trash by $B$. cinerea at flowering and postflowering, should contribute to

†Corresponding author: V. Rossi; vittorio.rossi@unicatt.it

The author(s) declare no conflict of interest.

(C) 2019 The American Phytopathological Society control of BBR (Calvo-Garrido et al. 2014; González-Domínguez et al. 2015). Reduction of available bunch trash has been explored by removal of floral debris from clusters either at early or late fruit set using compressed air or leaf blowers (Wolf et al. 1997) and fungicide sprays (González-Domínguez et al. 2015) and the application of biocontrol agents and other natural products (Calvo-Garrido et al. 2014; Pertot et al. 2017) has been investigated to reduce colonization of bunch trash by $B$. cinerea.

Evaluation of the effectiveness of these interventions requires methods to quantify the colonization of bunch trash by $B$. cinerea and the subsequent production of spores. Traditionally, $B$. cinerea colonization of bunch trash has been quantified by plating on selective media (Abdelwahab and Younis 2012; Edwards and Seddon 2001) or by microscopic assessment (Calvo-Garrido et al. 2014). Sporulation on bunch trash was measured using a sporulation index on a 0 to 5 scale (Calvo-Garrido et al. 2014) or by microscope counts of conidia (Jaspers et al. 2013; Keller et al. 2003; Mundy et al. 2012; Nair et al. 1995). As alternatives to traditional methods, molecular tools may offer rapid, specific, and accurate estimation of the quantity of $B$. cinerea in bunch trash (Abdelwahab and Younis 2012; Diguta et al. 2010).

A direct polymerase chain reaction (PCR) assay has been described for the detection of $B$. cinerea in pea-sized berries and receptacles (Gindro et al. 2005). Real-time quantitative PCR (qPCR) assays have been developed for the detection and quantification of $B$. cinerea inoculum (conidia and/or mycelium) from air samples and the surface of ripe berries (Carisse et al. 2014; Diguta et al. 2010) and for the quantification of the colonization of $B$. cinerea in developing grape berries and receptacles (CadleDavidson 2008; Saito et al. 2013), grape stamens, and ripe berries (Celik et al. 2009; Hill et al. 2014; Sanzani et al. 2012).

The objectives of the current study were as follows: (i) to optimize a hydrolysis probe-based qPCR assay for the quantification of $B$. cinerea DNA in bunch trash; (ii) to investigate the relationships between the quantity of $B$. cinerea DNA measured by qPCR, and the colonization measured based on the plating method, 
and as measured by sporulation potential (SP) of bunch trash using microscope counts of conidia; and (iii) to evaluate the qPCR assay under vineyard conditions.

\section{MATERIALS AND METHODS}

Real-time qPCR optimization. Fungal isolates. Strains of $B$. cinerea belonging to the transposon genotypes transposa (isolate $213 \mathrm{~T}$ ) and vacuma (isolate $351 \mathrm{~V}$ ) (Ciliberti et al. 2016) and other fungal isolates (Table 1) were obtained from the culture collection of the Department of Sustainable Crop Production of the Università Cattolica del Sacro Cuore (UCSC) in Piacenza, Italy. The biotrophic pathogens Plasmopara viticola and Erysiphe necator were collected from symptomatic leaves (cultivar Merlot) in the vineyard of UCSC in 2017 and 2018 and were maintained on containergrown grape plants (Merlot) in a greenhouse kept at a temperature of $24 \pm 3^{\circ} \mathrm{C}$ with a 12 -h photoperiod.

Plant material. Bunch trash was obtained from plants grown in a greenhouse to minimize natural colonization by $B$. cinerea. Woody cuttings were collected in winter from an experimental vineyard ( $V$. vinifera 'Merlot') at UCSC. Cuttings were grown in a greenhouse maintained at $24 \pm 3^{\circ} \mathrm{C}$ with a 12-h photoperiod, and flowers were obtained following the technique of Mullins and Rajasekaran (1981). At full flowering, bunch trash was collected by gently shaking the inflorescences inside paper bags. Bunch trash samples were desiccated at 35 to $40^{\circ} \mathrm{C}$ for $72 \mathrm{~h}$, and the dry weights were determined. Samples were stored at room temperature until use.

DNA extraction. Genomic DNA was obtained from 15 fungal species (Table 1) and bunch trash samples. Except in the case of $P$. viticola and E. necator, genomic DNA was extracted from fresh mycelium (obtained by scraping the surface of 10-day-old-colonies grown on potato dextrose agar [PDA], at $20^{\circ} \mathrm{C}$ with a 18 -h photoperiod). DNA of $P$. viticola and E. necator was obtained from leaf discs with lesions showing abundant and fresh sporulation (100 mg of leaf material). In brief, mycelium (100 mg fresh weight) or bunch trash (100 mg dry weight) was placed in $2-\mathrm{ml}$ microcentrifuge tubes containing $100 \mathrm{mg}$ of glass sand (425 to $600 \mu \mathrm{m}$ in diameter), two glass beads ( $5 \mathrm{~mm}$ in diameter), and $500 \mu \mathrm{l}$ of cetyl trimethylammonium bromide (CTAB) extraction buffer (2\% CTAB, $100 \mathrm{mM}$ of Tris- $\mathrm{HCl}, \mathrm{pH} 8.0,20 \mathrm{mM}$ of ethylenediaminetetraacetic acid, $1.4 \mathrm{M}$ of $\mathrm{NaCl}$, and $1 \%$ polyvinylpyrrolidone). The tubes were placed in a Mixer Mill MM200 (Retsch $\mathrm{GmbH}$, Haan, Germany) for $1 \mathrm{~min}$ at 30 cycles/s. Subsequently, a CTAB DNA extraction procedure was conducted as described by Saito et al. (2013). The yield and purity of the extracted DNA was determined using a NanoDrop 2000 spectrophotometer (Thermo Fisher Scientific Inc., Waltham, MA). The extracts were adjusted to $10 \mathrm{ng} / \mu \mathrm{l}$ of DNA for fungal samples and to $20 \mathrm{ng} / \mu \mathrm{l}$ of DNA for bunch trash samples.

Primers and hydrolysis probes. The $\mathrm{qPCR}$ assay was based on two specific primers and a hydrolysis probe $(\mathrm{Bc} 3)$ designed to target the intergenic spacer region (IGS) of the nuclear ribosomal DNA (Suarez et al. 2005) of $B$. cinerea. To normalize the quantification DNA of $B$. cinerea in plant tissues, two specific primers and a hydrolysis probe (Res) designed to target the $V$. vinifera resveratrol synthase gene I (Valsesia et al. 2005) were used as an internal control, with the fluorescent reporter JOE as a substitute for FAM (6-carboxyfluorescein). The sequences for $\mathrm{Bc} 3$ were as follows: 5'-GCT GTA ATT TCA ATG TGC AGA ATC C-3' (forward [Bc3F]), 5'-GGA GCA ACA ATT AAT CGC ATT TC-3' (reverse [Bc3R]); and 5' -6-FAM-TCACCT TGC AAT GAG TGG-BHQ-1-3' (probe [Bc3P]). The sequences for Res were as follows: 5'-CGA GGA ATT TAG AAA CGC TCA AC-3' (forward [ResF]), 5'-GCT GTG CCA ATG GCT AGG A-3' (reverse [ResR]), and 5'-JOE-TGC CAA GGG TCC GGC CAC C-TAMRA-3' (probe [ResP]).

Singleplex and duplex reactions. Singleplex reaction mixtures contained the 1× QuantiTect Multiplex PCR Kit (Qiagen, Milano,
Italy), $150 \mathrm{nM}$ of probe (Bc3P or ResP), $500 \mathrm{nM}$ of each primer $(\mathrm{Bc} 3 \mathrm{~F} / \mathrm{R}$ or $\mathrm{ResF} / \mathrm{R})$, and $2 \mu \mathrm{l}$ of DNA template in a final volume of $10 \mu$ l. Duplex reaction mixtures contained the $1 \times$ QuantiTect Multiplex PCR Kit, $150 \mathrm{nM}$ of the $V$. vinifera probe ResP, $150 \mathrm{nM}$ of the $B$. cinerea probe $\mathrm{Bc} 3 \mathrm{P}, 100 \mathrm{nM}$ of each $V$. vinifera primer $(\mathrm{Res} \mathrm{F} /$ $\mathrm{R}), 500 \mathrm{nM}$ of each $B$. cinerea primer $(\mathrm{Bc} 3 \mathrm{~F} / \mathrm{R})$, and $2 \mu \mathrm{l}$ of DNA template in a final volume of $10 \mu \mathrm{l}$. Both singleplex and duplex assays were performed using an Applied Biosystems 7300 RealTime PCR System (Thermo Fisher Scientific Inc.) with an initial incubation at $95^{\circ} \mathrm{C}$ for $15 \mathrm{~min}$ followed by 40 cycles of $95^{\circ} \mathrm{C}$ for $15 \mathrm{~s}$ and $60^{\circ} \mathrm{C}$ for $45 \mathrm{~s}$.

Specificity. The specificity of the qPCR assay for the detection of $B$. cinerea in grape tissue was determined in a test that included the most common grape pathogens and other fungal species frequently found in grapevines and in air samples (Table 1); several of these species were not assessed by Suarez et al. (2005).

Standard curves, calibration DNA, and qPCR optimization. Standard curves were obtained from two singleplex qPCR assays: the Res assay with DNA of $V$. vinifera from bunch trash as a template in a 10 -fold dilution series (from 20 to $0.02 \mathrm{ng} / \mu \mathrm{l}$ ), and the Bc3 assay with DNA of $B$. cinere $a$ extracted from the mycelium (isolate 213T, Ciliberti et al. 2016) as a template in a 10-fold dilution series (from 10 to $0.001 \mathrm{ng} / \mu \mathrm{l})$. Singleplex qPCR assays were carried out twice; for each assay, each dilution was replicated three times. A water control was included in triplicate in each assay. To detect any potential inhibition of amplification of $B$. cinerea DNA by $V$. vinifera DNA, duplex qPCR assays were performed with DNA of $B$. cinerea mixed with DNA of $V$. vinifera, following the approach described by Saito et al. (2013). In brief, $1 \mu \mathrm{l}$ from each of the previously described DNA dilutions for $B$. cinerea was mixed with $1 \mu \mathrm{l}$ of $V$. vinifera DNA ( $20 \mathrm{ng} / \mu \mathrm{l})$, yielding a 10-fold dilution series from 1:2 to 1:20,000 w/w of B. cinerea/V. vinifera DNA. The duplex qPCR assay was performed twice with three replicates for each dilution. Standard curves of both singleplex and duplex qPCR assays were produced by linear regression, and the coefficient of determination $\left(R^{2}\right)$ was calculated. The amplification efficiency $(\mathrm{E})$ of all assays was determined from the slope of the standard curves (Bustin et al. 2009). To allow comparisons among the results of different tests, all duplex qPCR plates contained a calibration DNA template in triplicate consisting of $1 \mathrm{ng} / \mu \mathrm{l}$ of $B$. cinerea DNA diluted in $20 \mathrm{ng} / \mu \mathrm{l}$ of $V$. vinifera DNA $(1: 20$, wt/wt, of $B$. cinereal $V$. vinifera DNA).

Colonization coefficient. To quantify DNA of $B$. cinerea in bunch trash, known numbers of conidia or known weights of fresh mycelium were added to noncolonized bunch trash; the trash was obtained from the plants grown under isolation, and the absence

TABLE 1. List of isolates screened during specificity tests of the real-time quantitative polymerase chain reaction (qPCR) assay used to quantify Botrytis cinerea in grape bunch trash

\begin{tabular}{lcc}
\hline Genus and species & Isolate code & qPCR result $^{\mathrm{a}}$ \\
\hline Alternaria alternata & 5 & - \\
Alternaria sp. & 23 & - \\
Aspergillus flavus & 4 & - \\
Aspergillus niger & $\mathrm{A} 1$ & - \\
B. cinerea & $213 \mathrm{~T}$ and 351V & + \\
Erysiphe necator & FP 2017 and FP 2018 & - \\
Guignardia bidwellii & Q15 and C14 & - \\
Monilia laxa & 11 & - \\
Penicillium sp. & 2 & - \\
Phomopsis viticola & Pho-1 and Pho-6 & - \\
Plasmopara viticola & FP 2017 and FP 2018 & - \\
Rhizopus sp. & 26 & - \\
R. stolonifer & MUCL38013 & - \\
Sclerotinia sclerotiorum & 22 & - \\
Stemphylium sp. & 14 & -
\end{tabular}

a Minus signs indicate not amplified, and the plus sign indicates amplified.

b $\mathrm{FP}=$ field population and year of collection. 
of $B$. cinerea was confirmed by a $\mathrm{Bc} 3$ qPCR assay. Conidial suspensions $(10 \mu \mathrm{l}$ containing from 1 to 1,250 conidia/ $\mu \mathrm{l})$ or fresh mycelia $(5.0,1.0,0.5$, or $0.1 \mathrm{mg})$ were added to $0.1-\mathrm{g}$ bunch trash samples in 2-ml microcentrifuge tubes. The total DNA from two biological replicates of each sample was then extracted as described earlier; noncolonized bunch trash without the addition of $B$. cinerea was used as a negative control. Duplex qPCR assays were performed twice for each experiment with two technical replicates of each template DNA. A water control and calibration DNA were included in each assay.

The quantification of DNA of B. cinerea in the presence of DNA of $V$. vinifera was expressed in terms of a colonization coefficient (CC), which was the ratio between DNA concentrations of $B$. cinerea and $V$. vinifera, corrected by a correction coefficient ( $\triangle \mathrm{CC}$ ) (Gusberti et al. 2012). CC values were calculated as follows:

$$
\mathrm{CC}=\mathrm{DNA}_{B . \text { cinerea }} / \mathrm{DNA}_{V . \text { vinifera }}+\Delta \mathrm{CC}
$$

DNA amounts were obtained by transforming the quantification cycles $(\mathrm{Cq})$ values of both targets ( $B$. cinerea and $V$. vinifera) according to the standard curves obtained from the serial dilution assays (Table 2) as follows:

$$
\operatorname{DNA}(\mathrm{ng} / \mu \mathrm{l})=10^{[\text {(Cq value }-\mathrm{y} \text {-axis intercept }) / \text { slope }]}
$$

$\Delta \mathrm{CC}$ was calculated as the difference between the average $\mathrm{CC}$ value of calibrator DNA calculated in the standard curve $\left(\mathrm{CC}_{\mathrm{St}}=5.15\right)$ and the average $\mathrm{CC}$ value of calibrator DNA of the assay $\left(\mathrm{CC}_{\mathrm{A}}\right)$ (i.e., $\Delta \mathrm{CC}=\mathrm{CC}_{\mathrm{St}}-\mathrm{CC}_{\mathrm{A}}$ ).

Evaluation of the qPCR method with inoculated bunch trash. Plant material. Bunch trash samples were collected in 2017 in a vineyard located in Castell' Arquato (CA) in the EmiliaRomagna region of Northern Italy $\left(44^{\circ} 51^{\prime} 26.1^{\prime \prime} \mathrm{N} 9^{\circ} 51^{\prime} 20.7^{\prime \prime} \mathrm{E}\right.$, $400 \mathrm{~m}$ above sea level). The CA vineyard was planted to Merlot, which is highly susceptible to $B$. cinerea (Bisiach et al. 1996; Corvi and Tullio 1980). The vines were 10 years old and were trained using the Guyot system. The within and between-row spacings were 1.0 and $2.3 \mathrm{~m}$, respectively. The vineyard was managed following an integrated pest management (IPM) program, with between-row grass, branches pruned to 10 to 12 buds per cane, and no irrigation. Vines were not treated for control of $B$. cinerea. At full flowering (stage 65 of Lorenz et al. 1994), bunch trash was collected from the vines by gently shaking grape bunches inside paper bags. Bunch trash samples were transported to the laboratory and were immediately desiccated at 35 to $40^{\circ} \mathrm{C}$ for $72 \mathrm{~h}$, and the dry weights were determined. Samples were stored at room temperature.

Preparation of inoculum of $\mathrm{B}$. cinerea and inoculation of bunch trash. Conidia of B. cinerea (isolate 213T) were obtained from 10day-old cultures grown on PDA. The conidial suspensions were prepared by flooding the dishes with sterile-distilled water and gently scraping the agar surface with a sterile rod. The suspension was filtered through two layers of autoclaved gauze and quantified using a hemocytometer. The inoculum concentration was adjusted to $10^{5}$ conidia/ml.

The samples of bunch trash $(0.1 \mathrm{~g})$ collected in the vineyard were placed on autoclaved filter paper discs in Petri dishes $(60 \mathrm{~mm}$ in diameter) and inoculated with $1 \mathrm{ml}$ of the conidial suspension of $B$. cinerea by using a micropipette. The samples were incubated at $20^{\circ} \mathrm{C}$ for $18 \mathrm{~h}$ in the dark to favor conidial germination and bunch trash colonization. The colonized samples were dried in a laminar flow hood at room temperature for $2 \mathrm{~h}$. Bunch trash samples with different degrees of colonization by $B$. cinerea $(0,25,50,75$, and $100 \%$ ) were obtained by mixing colonized and noncolonized bunch trash; for example, $75 \%$ colonization comprised $0.75 \mathrm{~g}$ of colonized bunch trash and $0.25 \mathrm{~g}$ of noncolonized bunch trash. Three replicate 1.0 -g samples were prepared for each colonization level.

Colonization of inoculated bunch trash as determined by $q P C R$. In the first assay, genomic DNA was extracted from $0.1 \mathrm{~g}$ of two replicate samples for each of the five bunch trash colonization levels. The extracted DNA was quantified by the duplex qPCR assay described earlier. A water control and calibration DNA were included in each assay. The quantity of DNA of $B$. cinerea in the presence of DNA of $V$. vinifera was expressed as a CC value.

Colonization of inoculated bunch trash as determined by plating. In the second assay, colonization of inoculated bunch trash by $B$. cinerea was quantified for three replicate samples of each of the five colonization levels by randomly and individually plating 50 pieces (stamens, aborted flowers, aborted berries, calyptras, tendrils, or leaf fragments) on PDA in Petri dishes $(90 \mathrm{~mm}$ in diameter). The dishes were incubated at $20^{\circ} \mathrm{C}$ with an 18 -h photoperiod for 3 days. The dishes were examined using a stereomicroscope, and the colonization rate (CR) was expressed as the percentage of pieces with characteristic grayish sporulation indicating the growth of $B$. cinerea.

$S P$ on inoculated bunch trash as determined by incubation and spore enumeration. In the third assay, the SP of $B$. cinerea on inoculated bunch trash was determined for three replicate samples $(0.05 \mathrm{~g}$ each $)$ at each colonization level. The bunch trash was placed on a disc of autoclaved filter paper in Petri dishes $(60 \mathrm{~mm}$ in diameter); sterile water ( $0.5 \mathrm{ml}$ per dish) was used to moisten the filter paper to maintain a saturated atmosphere. The dishes were sealed with Parafilm and incubated at $20^{\circ} \mathrm{C}$ with an $18-\mathrm{h}$ photoperiod to induce sporulation of $B$. cinerea. After 3 days of incubation, the bunch trash was suspended in $5 \mathrm{ml}$ of sterile water in a $15-\mathrm{ml}$ Falcon tube and mixed with a vortex apparatus for $10 \mathrm{~s}$. Conidia of $B$. cinerea were counted using a hemocytometer and expressed as the number of conidia per gram of dry bunch trash.

The bunch trash inoculation experiment and quantification of $B$ cinerea by qPCR, plating, and sporulation was performed three times.

Evaluation of the qPCR method with naturally inoculated bunch trash. Vineyards and treatments. Experiments were conducted in the CA vineyard and in two additional vineyards (designated MA and CO) located in Northern Italy in 2016 and 2017. The MA vineyard $\left(44^{\circ} 41^{\prime} 57^{\prime \prime} \mathrm{N} 12^{\circ} 19^{\prime} 66^{\prime \prime} \mathrm{E}\right.$, at sea level) is located in Mandriole in the Emilia-Romagna region, and the $\mathrm{CO}$ vineyard $\left(45^{\circ} 57^{\prime} 05^{\prime \prime} \mathrm{N} 13^{\circ} 27^{\prime} 19^{\prime \prime} \mathrm{E}, 1 \mathrm{~m}\right.$ at sea level) is located in Cormons in the Friuli-Venezia Giulia region of Italy. The MA vineyard was planted with cultivar Trebbiano Romagnolo, which were trained using the Casarsa system. Vines were 12 years old in 2016. The CO vineyard was planted with cultivar Merlot, which were trained using the Guyot system. Vines were 7 years old in 2016. Within- and between-row spacing in the MA and CO vineyards was 1.0 and $3.0 \mathrm{~m}$ and 0.8 and $2.4 \mathrm{~m}$, respectively. The MA vineyard was managed according to standard IPM practice in

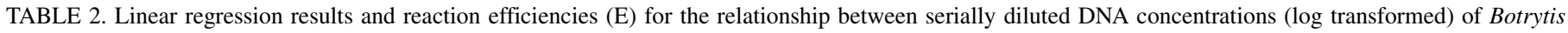

\begin{tabular}{|c|c|c|c|c|c|}
\hline qPCR assay & DNA template & Linear equation $^{\mathrm{a}}$ & $R^{2 \mathrm{~b}}$ & $P$ value ${ }^{\mathrm{c}}$ & $\mathrm{E}(\%)$ \\
\hline Singleplex Bc3 & B. cinerea & $y=-3.42 x+22.5$ & 0.99 & $<0.001$ & 96 \\
\hline Singleplex Res & V. vinifera & $y=-3.37 x+23.7$ & 0.99 & $<0.001$ & 98 \\
\hline Duplex Bc3/Res & B. cinerea $+V$. vinifera & $y=-3.56 x+24.0$ & 0.99 & $<0.001$ & 91 \\
\hline
\end{tabular}
cinerea and Vitis vinifera and corresponding $\mathrm{Cq}$ values obtained in singleplex and duplex quantitative polymerase chain reaction (qPCR) assays

${ }^{a}$ In the equations, $y$ refers to the $\mathrm{Cq}$ value, and $x$ refers to the DNA concentration.

${ }^{\mathrm{b}} R^{2}$ is the coefficient of determination of the regression.

c $P$ value indicates fit of the regression model. 
this region (Ministero delle politiche agricole alimentari, forestali e del turismo 2017), with between-row grass, vines pruned to 10 to 12 buds per cane, and emergency irrigation. The $\mathrm{CO}$ vineyard followed a conventional pest management strategy, with between-row grass, vines long pruned, and irrigation. Like Merlot in the $\mathrm{CA}$ and $\mathrm{CO}$ vineyards, Trebbiano Romagnolo in the MA vineyard is highly sensitive to B. cinerea (Bisiach et al. 1996; Corvi and Tullio 1980).

In each of the three vineyards, fungicide treatments were applied to obtain a range of colonization of bunch trash by B. cinerea. There were two treatments: (i) nontreated control (NT), and (ii) fungicide applied at full flowering (stage 65) (T). Treatments were arranged in a complete randomized block design with four replicate plots per treatment and with six plants per plot. The $\mathrm{T}$ treatment was a commercial mixture of fludioxonil $(25 \%)$ and cyprodinil $(37.5 \%)$ (Switch; Syngenta Crop Protection) applied at $0.8 \mathrm{~g} /$ liter of water until run-off using a 15-liter Elettroplus knapsack sprayer (Davide e Luigi Volpi S.p.a, Casalromano, Italy). Seven days after the treatment, bunch trash was collected from five random bunches per plot; these were combined to yield four replicate trash bunches per treatment.

Colonization of naturally inoculated bunch trash as determined by $q P C R$. Genomic DNA in the naturally inoculated bunch trash was extracted from each of the four replicate samples per treatment $(0.1 \mathrm{~g}$ each) and was quantified by the duplex qPCR assay as described previously; there were two technical replicates of each template DNA. A water control and calibration DNA were included in each assay. The quantities of DNA of $B$. cinerea in the presence of DNA of $V$. vinifera were expressed as $C C$ values.

$S P$ on naturally inoculated bunch trash as determined by incubation and spore enumeration. The SP of B. cinerea in the naturally inoculated bunch trash was determined for each of the four replicate samples per treatment by wrapping the bunch trash in three layers of sterile filter paper to which $5 \mathrm{ml}$ of sterile water was added. The bunch trash in filter papers was sealed in polyethylene bags and incubated at $20^{\circ} \mathrm{C}$ for 5 days to induce sporulation in B. cinerea. Each sample of bunch trash was suspended in $15 \mathrm{ml}$ of sterile water in a 50-ml Falcon tube and vortexed. The conidia of $B$. cinerea were counted using a hemocytometer and the quantity was expressed as the number of conidia per gram of dry bunch trash.

Latent infection of naturally inoculated berries. The incidence of latent infection (ILI) of berries by $B$. cinerea was assessed in the three vineyards; 25 randomly selected, symptomless berries with the pedicel attached were collected at maturity (stage 89) in each replicate plot in both years. The berries were rinsed in tap water, surface sterilized by immersion for $1 \mathrm{~min}$ in a $30 \%$ sodium hypochlorite solution, and rinsed in sterile-distilled water for $1 \mathrm{~min}$. Berries were positioned individually over a metal grid that was placed in a sterile metal box, the bottom of which was covered with wet, sterile paper. The boxes were sealed in plastic bags to maintain a saturated atmosphere and were incubated for 7 days at $25^{\circ} \mathrm{C}$. The ILI was visually assessed as the percentage of berries showing typical sporulation of $B$. cinerea.

Data analysis. All statistical analyses were performed using SPSS (version 24; IBM SPSS Statistics, IBM Corp., Armonk, NY). For experiments with inoculated bunch trash, regression analysis was used to investigate the relationships between the number of conidia of $B$. cinerea or the quantity of mycelium added and the $\mathrm{CC}$, and between the $\mathrm{CC}$ and the $\mathrm{CR}$ of bunch trash or the SP on bunch trash. Both linear and nonlinear regression functions were used to explore these relationships:

$$
\begin{gathered}
\mathrm{Y}=a+b \mathrm{X} \\
\mathrm{Y}=\mathrm{Ymax} /(1+\exp [a-b \mathrm{X}])
\end{gathered}
$$

in which $a$ and $b$ are intercept and slope parameters and Ymax is the maximum value of $\mathrm{Y}$ in the experiments. The coefficient of determination $\left(R^{2}\right)$ was used to assess the strength of the relationship.

The data from the field experiment were subject to a factorial analysis of variance (ANOVA) to determine whether the quantity of DNA of $B$. cinerea in bunch trash, SP, and ILI were affected by the main effects of year (2016 and 2017), vineyard (CA, CO, and MA), treatment (T and NT), and their interactions. Prior to the ANOVA, the SP and ILI values were transformed by natural logarithm and arcsine functions, respectively, to ensure homogeneity of variances.

\section{RESULTS}

qPCR specificity and standard curves. The $\mathrm{Bc} 3$ probe/ primer set did not amplify the purified DNA of nontarget organisms but did amplify the purified DNA of $B$. cinerea (Table 1), demonstrating excellent specificity. In the singleplex qPCR reaction, the $B$. cinerea standard curve revealed a high reaction efficiency of $96 \%$, with a close relationship between the $\mathrm{Cq}$ values and the concentrations of DNA of $B$. cinerea obtained by dilution (Table 2). The Bc3 assay was able to amplify the lowest concentration of DNA of $B$. cinerea tested $(0.001 \mathrm{ng} / \mu \mathrm{l})$, demonstrating excellent sensitivity. In the duplex assay, the presence of grape DNA did not influence the sensitivity or coefficient of determination $\left(R^{2}\right.$ value), whereas the reaction efficiency of the Bc3 set was slightly reduced (Table 2). Similar results were obtained for the $V$. vinifera standard curve. The Res assay was able to amplify the lowest concentration of DNA of $V$. vinifera tested $(0.02 \mathrm{ng} / \mu \mathrm{l})$.

Evaluation of the qPCR assay using inoculated bunch trash. The CC values, which represented the quantity of DNA of $B$. cinerea detected in the presence of DNA of $V$. vinifera, were proportional to the number of conidia of $B$. cinerea added to the bunch trash (Fig. 1A) and to the quantity of mycelium of $B$. cinerea added to the bunch trash (Fig. 1B); the coefficients of determination indicated a strong linear regression between these variables $\left(R^{2}=\right.$ 0.92 and 0.97 , respectively). When the qPCR assay was used with bunch trash samples containing different proportions of noncolonized bunch trash and $B$. cinerea-colonized bunch trash, the $\mathrm{CC}$ values were strongly related to the percentage of bunch trash colonized by B. cinerea (Fig. 2A) $\left(R^{2}=0.93\right)$.

For inoculated bunch trash, the relationship between the $\mathrm{CC}$ value and the SP (as determined by incubation followed by microscopic counting of spores) and between the $\mathrm{CC}$ value and the $\mathrm{CR}$ (as determined by plating bunch trash pieces) was nonlinear (Fig. 2B and C). As CC increased, both SP and CR increased, but the rate of increase of SP and CR declined while CC continued to increase, resulting in a logistic relationship. The coefficients of determination indicated a strong relationship these variables $\left(R^{2}=0.92\right.$ and 0.97 , respectively).

Evaluation of the qPCR assay using naturally colonized bunch trash. The application of fungicides to control B. cinerea at flowering significantly reduced the CC (Fig. 3A), SP (Fig. 3B), and ILI on berries (Fig. 3C) $(P<0.001$ for all three variables; Table 3). Year had a significant effect on CC $(P \leq 0.001)$ : the degree of colonization of bunch trash was greater in 2017 (CC = $1.76 \pm 0.45)$ compared with colonization in $2016(\mathrm{CC}=0.23 \pm$ $0.09)$. Main effects of year also affected SP $(P<0.0001)$, but not ILI $(P=0.3)$. The main effect of vineyard affected SP $(P=0.02)$ and ILI $(P \leq 0.0001)$, but not CC $(P=0.7)$. In addition, SP was significantly influenced by the interaction of year $\times$ vineyard, and ILI by the interactions of year $x$ vineyard and year $\times$ treatment. Thus, the fungicide treatment reduced CC, SP, and ILI in all of the vineyards, irrespective of the initial degree of colonization of bunch trash by $B$. cinerea, the SP of the bunch trash, or the ILI of berries.

The interaction of year $\times$ vineyard $\times$ treatment had no significant effect on CC $(P=0.6), \operatorname{SP}(P=0.5)$, or ILI $(P=0.8)$, demonstrating that beyond the two-way interactions of main effects, there were no 
more complex associations in this study. These results indicate that the $\mathrm{CC}$ values reflected the reduction in sporulation of $B$. cinerea and the latent infection of berries caused by fungicide application at flowering. The qPCR method was able to detect differences between treated and nontreated plots in vineyards whether the CC of bunch trash by B. cinerea was $<0.1$ (Fig. 4A), $<3$ (Fig. 4B), or $<5$ (Fig. 4C).

\section{DISCUSSION}

We evaluated a qPCR assay for the quantification of $B$. cinerea DNA in grape bunch trash. The qPCR assay was based on the procedure developed by Saito et al. (2013), with a few adaptations regarding the handling of plant material prior to DNA extraction and the qPCR reaction mixture and conditions. The qPCR assay was compared with traditional mycological techniques for quantifying
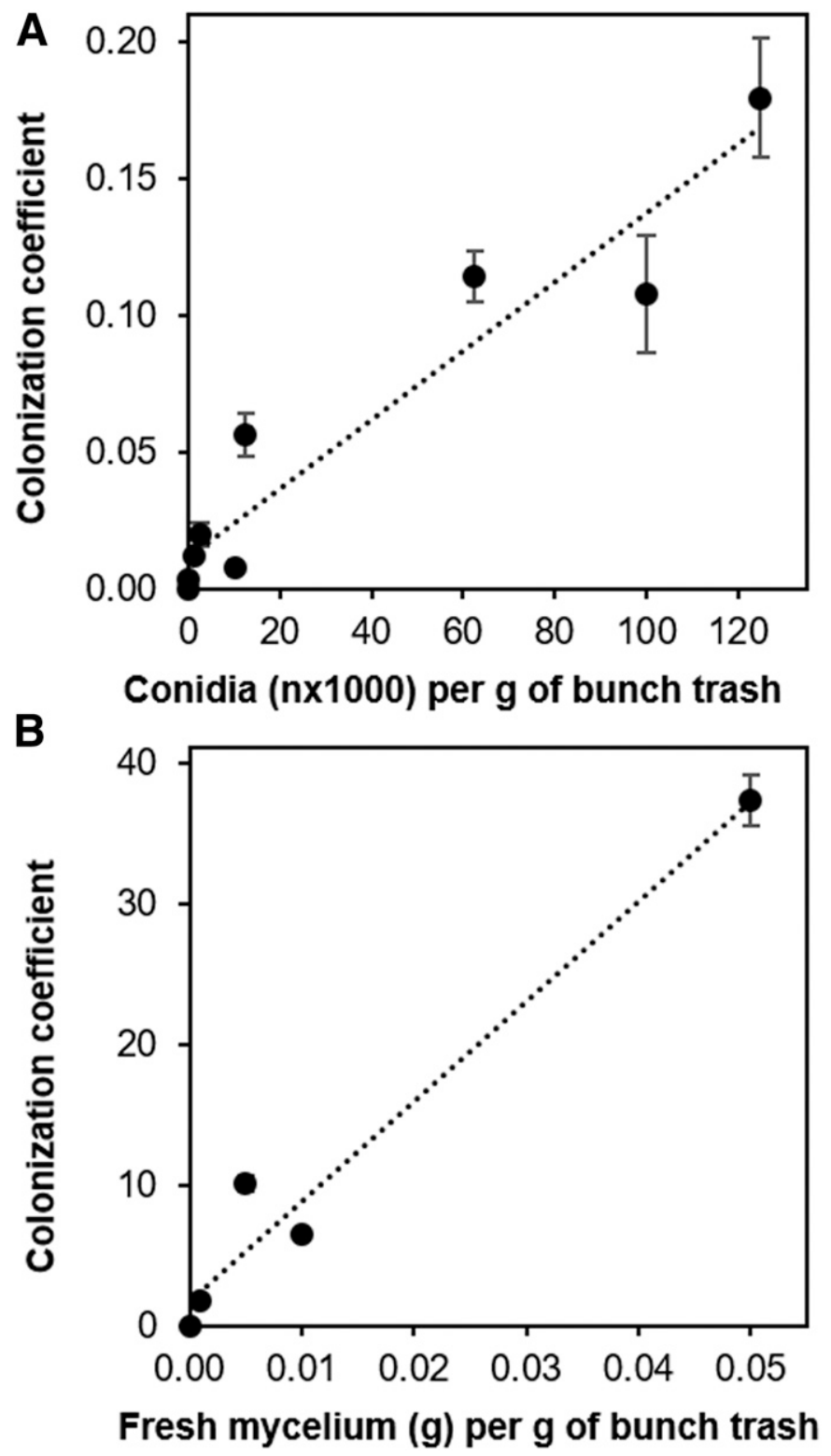

Fig. 1. Relationship between the colonization coefficient (the ratio between DNA concentrations of Botrytis cinerea and Vitis vinifera as determined by the quantitative polymerase chain reaction assay) and $\mathbf{A}$, the number of conidia added per gram of bunch trash, and $\mathbf{B}$, the weight of fresh mycelium added to the bunch trash in inoculation experiments. Markers indicate means, whiskers indicate standard errors, and dotted lines indicate the linear relationships. In A, $\mathrm{Y}=0.011+0.001 \mathrm{X}\left(R^{2}=0.92\right)$; and in $\mathrm{B}, \mathrm{Y}=1.75+710.74 \mathrm{X}\left(R^{2}=0.97\right)$.
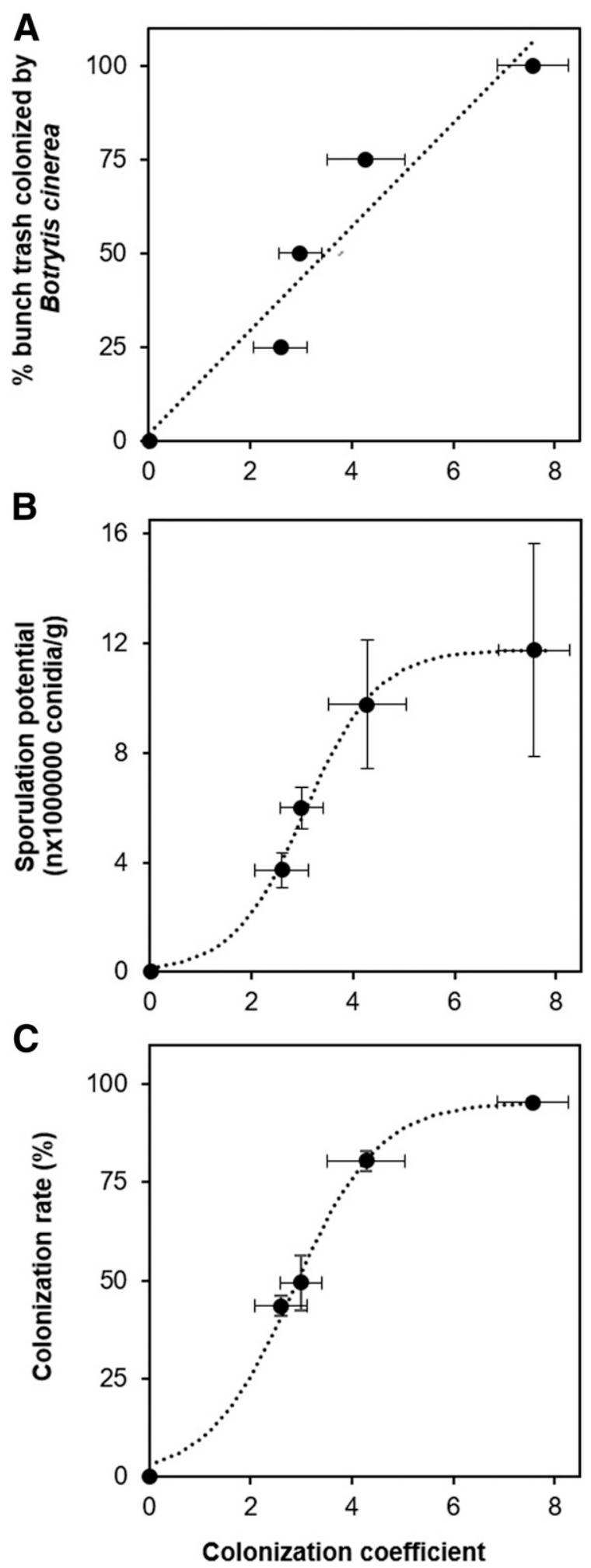

Fig. 2. Relationship between the colonization coefficient (the ratio between DNA concentrations of Botrytis cinerea and Vitis vinifera as determined by the quantitative polymerase chain reaction assay) of grape bunch trash and $\mathbf{A}$, the proportion (\%) of bunch trash that had been inoculated with and colonized by $B$. cinerea, $\mathbf{B}$, the sporulation potential of the bunch trash (expressed as the number of conidia produced per gram of bunch trash as determined by incubating the bunch trash in humid chambers), and $\mathbf{C}$, bunch trash colonization (expressed as the proportion [\%] of bunch trash pieces that were colonized by B. cinerea as determined by plating the bunch trash on potato dextrose agar). The grape bunch trash was inoculated with $B$. cinerea prior to being assayed for DNA of $B$. cinerea, sporulation potential, and colonization rate. Markers indicate means, whiskers indicate standard errors, and dotted lines indicate linear and nonlinear relationships. In $\mathrm{A}, \mathrm{Y}=1.79+13.81 \mathrm{X}\left(R^{2}=0.93\right)$; in $\mathrm{B}, \mathrm{Y}=95.5 /(1+\exp [3.38-1.19 \mathrm{X}])$ $\left(R^{2}=0.99\right)$; and in $\mathrm{C}, \mathrm{Y}=1.17 \mathrm{E}+07 /(1+\exp [4.31-1.41 \mathrm{X}])\left(R^{2}=0.99\right)$. 
B. cinerea based on colonization of and sporulation on bunch trash. Our results indicate that the qPCR assay and the CC calculation provide a sensitive and reliable method for quantifying colonization by $B$. cinerea of the trash materials (stamens, aborted flowers, aborted berries, calyptras, tendrils, and leaf pieces) remaining in grape bunches after flowering.

Colonized bunch trash serves as one of the primary sources of inoculum for the infection of ripening berries (Elmer and Michailides 2007; Holz et al. 2003; Nair et al. 1995). Thus, quantitative assessment of bunch trash colonization by $B$. cinerea is important for both research purposes and practical disease management, in order to make decisions regarding control of BBR. When the bunch trash has a low incidence of $B$. cinerea, subsequent development of BBR during berry ripening is likely to be low too (Keller et al. 2003; McClellan and Hewitt 1973; Pezet et al. 2003), and the number of fungicide applications can therefore be reduced (González-Domínguez et al. 2019). Visual assessment, plating on agar media, and microscope counts of spores have been commonly used to evaluate the colonization and the SP of B. cinerea in bunch trash (Abdelwahab and Younis 2012; Calvo-Garrido et al. 2014; Jaspers et al. 2013; Mundy et al. 2012); these traditional techniques are time-consuming and require expertise for the identification of $B$. cinerea colonies and/or conidia.

The results of Suarez et al. (2005) and those of our study indicate that the qPCR assay is highly specific to $B$. cinerea. We found that the $\mathrm{Bc} 3$ system amplified the DNA of different $B$. cinerea strains, including strains that belong to the transposon genotypes transposa (T) or vacuma (V) (Ciliberti et al. 2016), but did not amplify the DNA of phylogenetically related species (B. fabae, Monilia laxa, and Sclerotinia sclerotiorum). Also, the Bc3 system did not amplify the DNA of other common grapevine pathogens (E. necator, Guignardia bidwellii, Phomopsis viticola, and Plasmopara viticola) or of other fungal species frequently present in vineyards (Alternaria spp., Aspergillus spp., Penicillium spp., and Rhizopus spp.).

In addition to its specificity, the qPCR assay is also sensitive because the targeted IGS region is a multicopy gene (Bruns et al. 1991). The lowest DNA concentration of $B$. cinerea tested in this study $(0.001 \mathrm{ng} / \mu \mathrm{l})$ was amplified in both the singleplex Bc3 assay and the duplex Bc3/Res assay in the presence of grape DNA, which is consistent with the results obtained by Saito et al. (2013) and Hill et al. (2014). Suarez et al. (2005) showed that the Bc3 assay is able to detect DNA concentrations as low as $20 \mathrm{fg} / \mu \mathrm{l}$.

The results of the qPCR assay were used to calculate the CC (i.e., the ratio of the pathogen and host DNA concentrations) (Gusberti et al. 2012). In duplex qPCR analyses, researchers have described several methods for calculating the quantity of DNA of a pathogen in host tissue. To account for variation among samples and qPCR runs in terms of tissue weight, pipetting volumes, and efficiencies of DNA extraction and amplification, these methods account for amounts of host plant DNA in order to provide internal normalization. Sanzani et al. (2012), for example, normalized the DNA concentration of $B$. cinerea according to the quantity of host DNA by using a host DNA correction factor for each grape sample. Valsesia et al. (2005) developed the infection coefficient (IC), which is based on the ratio between $\mathrm{Cq}$ values of the pathogen and host generated by the qPCR assay. The IC approach was also used to determine the pathogen coefficient (PC) of B. cinerea in grape berries and receptacles (Hill et al. 2014; Saito et al. 2013). In a preliminary analysis, we found that the PC values based on the current data increased as the number of $B$. cinerea conidia or quantity of mycelium added to bunch trash increased. However, in the inoculation experiments, the PC values did not increase linearly as the level of colonization increased from 0 to $100 \%$ (data not shown). These preliminary results generally agree with those of Saito et al. (2013) and Hill et al. (2014), who found that the accuracy of PC decreased as the
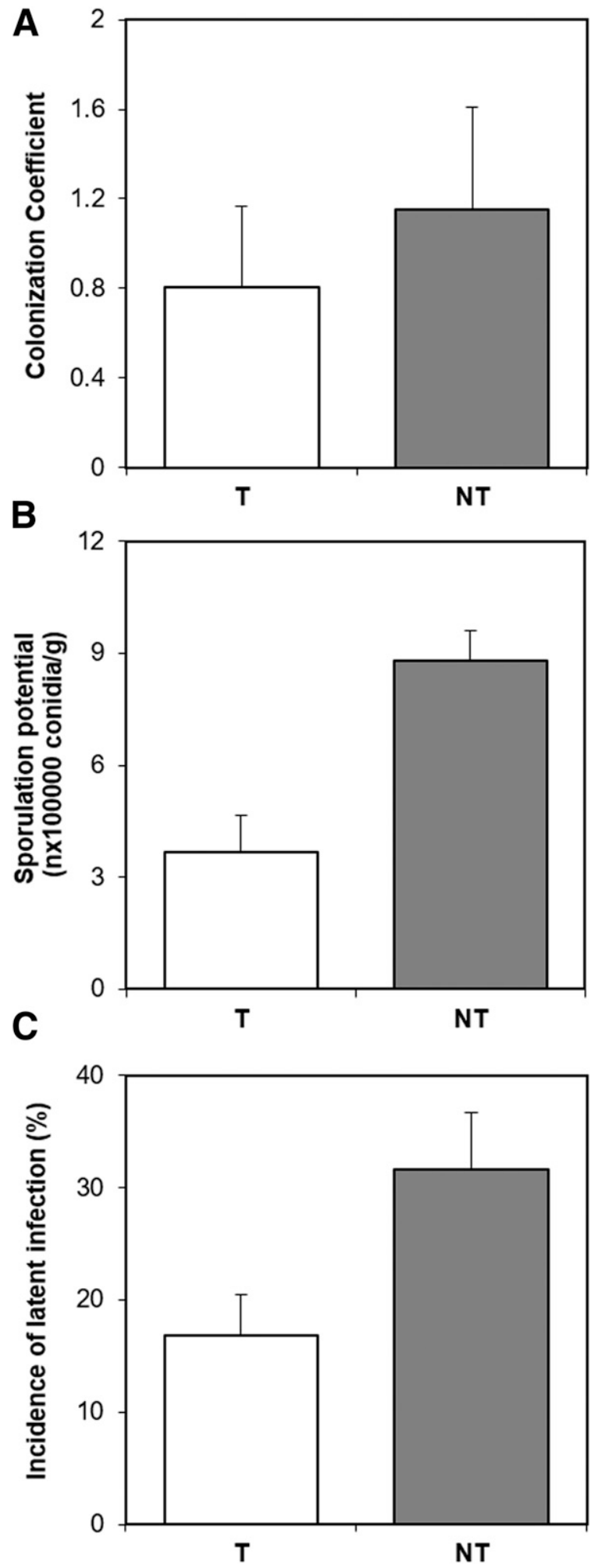

Fig. 3. Effect of the application of fungicides (a mixture of fludioxonil [25\%] and cyprodinil [37.5\%]) to control Botrytis cinerea at flowering on $\mathbf{A}$, the colonization coefficient (the ratio between DNA concentrations of $B$. cinerea and Vitis vinifera in naturally colonized grape bunch trash as determined by the quantitative polymerase chain reaction assay), $\mathbf{B}$, the sporulation potential of bunch trash (expressed as the number of conidia produced per gram of naturally inoculated bunch trash after incubation in humid chambers), and $\mathbf{C}$, the incidence of grape berries with latent infection (expressed as the percentage of berries showing the characteristic sporulation of B. cinerea). Bars indicate means of treated (T) and nontreated (NT) plots in three vineyards from 2 years of data, and whiskers indicate standard errors $(n=24$; three vineyards, 2 years, four replicates). In each panel, means are significantly different $(P<0.001)$. 
severity of BBR increased. For this reason, we used the $\mathrm{CC}$ rather than the PC in the current study.

The results of the qPCR assay were comparable to those obtained with the traditional methods used to estimate $B$. cinerea colonization of and sporulation on bunch trash. Therefore, the qPCR assay described in this work is a valuable alternative to the traditional methods. Traditional methods and $\mathrm{qPCR}$ require expertise in mycology and molecular biology, respectively. The traditional methods are time-consuming, whereas the qPCR assay takes 3 to 4 $\mathrm{h}$. They also have limitations that potentially reduce their accuracy. Plating of trash pieces on agar can lead to the growth of other fastgrowing fungal species that may result in lower estimates of the number of $B$. cinerea colonies, while the accurate enumeration of spores from incubated trash in humid chambers is dependent on the operator's expertise to correctly identify conidia. The qPCR assay, on the contrary, provides sensitive and specific results.

The validity of the qPCR assay was confirmed in the field using bunch trash naturally colonized with $B$. cinerea that had been treated or not treated with fungicides during flowering. In the field, the $\mathrm{CC}$ values were consistent with the reduction of the SP caused by fungicide treatment and revealed differences between fungicidetreated plants and nontreated plants under different environmental conditions, even in situations where the incidence of colonization of bunch trash by $B$. cinerea was very low. The $\mathrm{CC}$ values of bunch trash were also consistent with the reduction in the ILI of berries caused by fungicide application at flowering. Although the latter result requires confirmation, it suggests that when the colonization of bunch trash is low, the ILI is also low as a result of unfavorable conditions for reproduction of $B$. cinerea during flowering.

In conclusion, the qPCR methodology described here is a sensitive, specific, and reliable tool for quantifying $B$. cinerea in bunch trash in vineyards. The qPCR assay can be used as an alternative to traditional methods for the quantification of $B$. cinerea during the early-season period (as an indicator of inoculum potential) and thus BBR severity at harvest; it can also be used as a tool in other epidemiological studies and to determine the effect of disease management methods on the reduction of inoculum of B. cinerea.

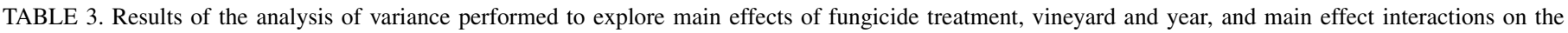
quantity of DNA of Botrytis cinerea in bunch trash, the sporulation potential of bunch trash, and the incidence of grape berries with latent infection ${ }^{\mathrm{a}}$

\begin{tabular}{|c|c|c|c|c|c|c|c|}
\hline \multirow[b]{2}{*}{ Main effects and interactions } & \multirow[b]{2}{*}{$d f^{b}$} & \multicolumn{2}{|c|}{ Colonization coefficient } & \multicolumn{2}{|c|}{ Sporulation potential } & \multicolumn{2}{|c|}{$\begin{array}{c}\text { Incidence of latent } \\
\text { infections }\end{array}$} \\
\hline & & $F$ value & $P$ value & $F$ value & $P$ value & $F$ value & $P$ value \\
\hline 1. Year & 1 & 17.4 & $<0.001$ & $2,309.3$ & $<0.001$ & 1.3 & 0.3 \\
\hline 2. Vineyard & 2 & 0.4 & 0.7 & 4.4 & 0.02 & 78.5 & $<0.001$ \\
\hline 3. Treatment & 1 & 17.8 & $<0.001$ & 50.6 & $<0.001$ & 54.9 & $<0.001$ \\
\hline $1 \times 2$ & 2 & 1.7 & 0.2 & 21.8 & $<0.001$ & 8.4 & 0.001 \\
\hline $1 \times 3$ & 1 & 2.7 & 0.08 & 1.8 & 0.2 & 4.8 & 0.04 \\
\hline $2 \times 3$ & 2 & 0.2 & 0.8 & 2.1 & 0.1 & 2.6 & 0.09 \\
\hline $1 \times 2 \times 3$ & 2 & 0.6 & 0.6 & 0.8 & 0.5 & 0.2 & 0.8 \\
\hline
\end{tabular}

a The quantity of DNA of $B$. cinerea in bunch trash was defined as the colonization coefficient determined by the quantitative polymerase chain reaction assay, the sporulation potential of bunch trash was defined as the natural logarithm of the number of conidia produced per gram of naturally inoculated bunch trash after incubation in humid chambers, and the incidence of grape berries with latent infection was defined as the arcsin transformation of the percentage of berries showing the characteristic sporulation of B. cinerea. Samples were collected from a field experiment comparing grape bunches either treated with a commercial mixture of fludioxonil (25\%) and cyprodinil (37.5\%) (Switch; Syngenta Crop Protection) applied at 0.8 g/liter of water or not treated, with the experiment conducted in 2016 and 2017 in three vineyards in Italy.

$\mathrm{b}$ df $=$ degrees of freedom.
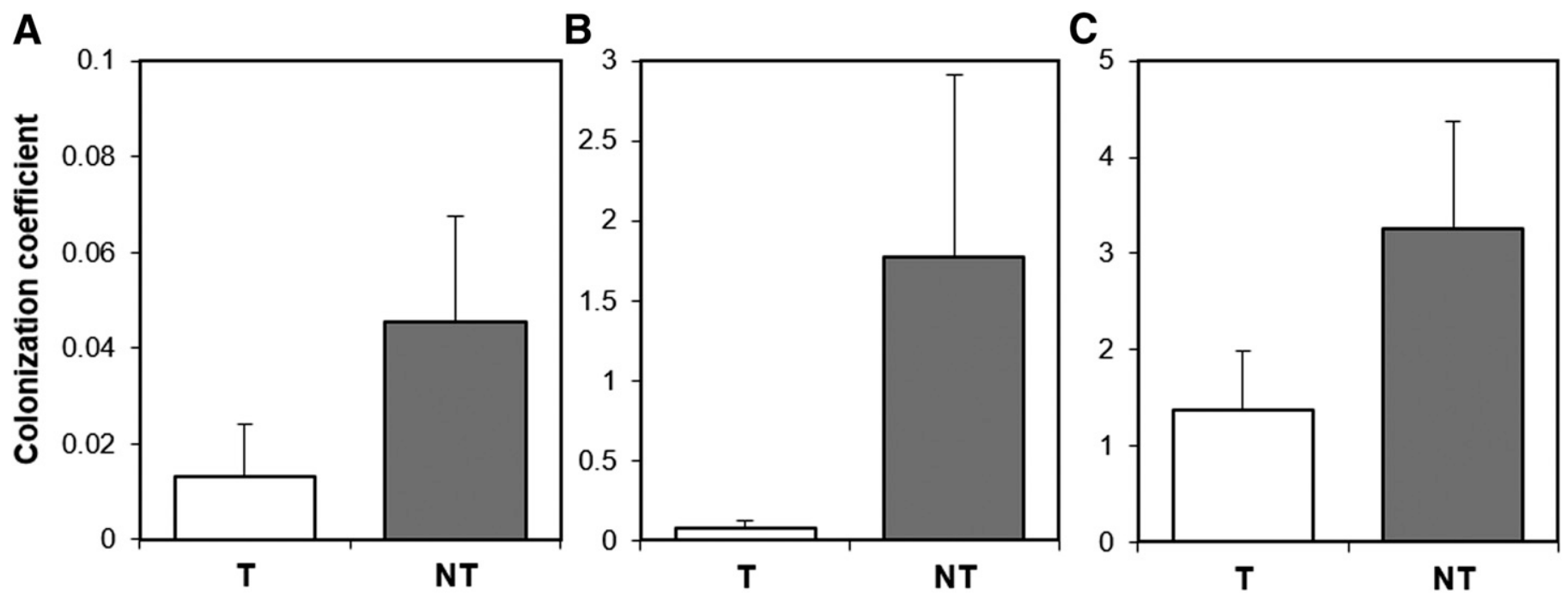

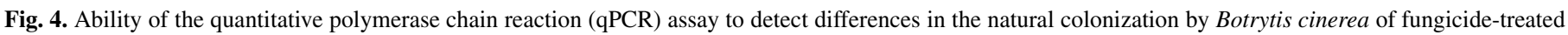

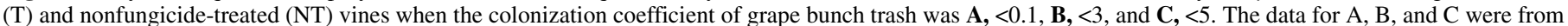

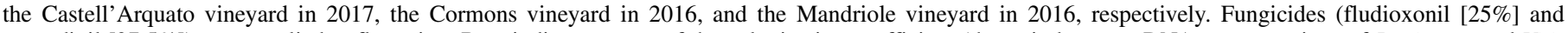

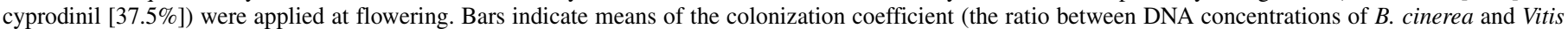

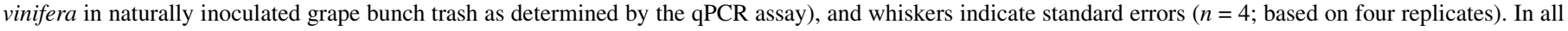
panels, treatments are significantly different at $P<0.001$. The interaction of year $\times$ vineyard $\times$ treatment was not significant $(P=0.6)$. 


\section{ACKNOWLEDGMENTS}

G. Fedele conducted this study within the Doctoral School of the AgroFood System (Agrisystem) at UCSC.

\section{LITERATURE CITED}

Abdelwahab, H., and Younis, R. A. 2012. Early detection of gray mold in grape using conventional and molecular methods. Afr. J. Biotechnol. 11: 15241-15245.

Bisiach, M., Zerbetto, F., and Cortesi, P. 1996. Attività fungicida della miscela cyprodinil + fludioxonil contro Botrytis cinerea su vite da vino. ATTI Giornate Fitopatologiche 2:363-368.

Bruns, T. D., White, T. J., and Taylor, J. W. 1991. Fungal molecular systematics. Annu. Rev. Ecol. Syst. 22:525-564.

Bustin, S. A., Benes, V., Garson, J. A., Hellemans, J., Huggett, J., Kubista, M., Mueller, R., Nolan, T., Pfaffl, M. W., Shipley, G. L., Vandesompele, J., and Wittwer, C. T. 2009. The MIQE guidelines: Minimum information for publication of quantitative real-time PCR experiments. Clin. Chem. 55:611-622.

Cadle-Davidson, L. 2008. Monitoring pathogenesis of natural Botrytis cinerea infections in developing grape berries. Am. J. Enol. Vitic. 59:387-395.

Calvo-Garrido, C., Usall, J., Viñas, I., Elmer, P. A., Cases, E., and Teixidó, N. 2014. Potential secondary inoculum sources of Botrytis cinerea and their influence on bunch rot development in dry Mediterranean climate vineyards. Pest Manag. Sci. 70:922-930.

Carisse, O., Tremblay, D. M., and Lefebvre, A. 2014. Comparison of Botrytis cinerea airborne inoculum progress curves from raspberry, strawberry and grape plantings. Plant Pathol. 63:983-993.

Celik, M., Kalpulov, T., Zutahy, Y., Ish-shalom, S., Lurie, S., and Lichter, A. 2009. Quantitative and qualitative analysis of Botrytis inoculated on table grapes by qPCR and antibodies. Postharvest Biol. Technol. 52:235-239.

Ciliberti, N., Fermaud, M., Languasco, L., and Rossi, V. 2015a. Influence of fungal strain, temperature, and wetness duration on infection of grapevine inflorescences and young berry clusters by Botrytis cinerea. Phytopathology 105:325-333.

Ciliberti, N., Fermaud, M., Roudet, J., Languasco, L., and Rossi, V. 2016. Environmental effects on the production of Botrytis cinerea conidia on different media, grape bunch trash, and mature berries. Aust. J. Grape Wine Res. 22:262-270.

Ciliberti, N., Fermaud, M., Roudet, J., and Rossi, V. 2015b. Environmental conditions affect Botrytis cinerea infection of mature grape berries more than the strain or transposon genotype. Phytopathology 105:1090-1096.

Corvi, F., and Tullio, V. 1980. Un biennio di prove di lotta contro la muffa grigia dell'uva (Botrytis cinerea Pers.) nelle marche (II contributo). Giornate Fitopatol. 2:553-560.

Diguta, C. F., Rousseaux, S., Weidmann, S., Bretin, N., Vincent, B., Guilloux-Benatier, M., and Alexandre, H. 2010. Development of a qPCR assay for specific quantification of Botrytis cinerea on grapes. FEMS Microbiol. Lett. 313:81-87.

Edwards, S. G., and Seddon, B. 2001. Selective media for the specific isolation and enumeration of Botrytis cinerea conidia. Lett. Appl. Microbiol. 32:63-66.

Elad, Y., Williamson, B., Tudzynski, P., and Delen, N, eds. 2007. Botrytis: Biology, Pathology and Control. Springer, Dordrecht, The Netherlands

Elmer, P. A. G., and Michailides, T. J. 2007. Epidemiology of Botrytis cinerea in orchard and vine crops. Pages 243-272 in: Botrytis: Biology, Pathology and Control. Y. Elad, B. Williamson, P. Tudzynski, and N. Delen, eds. Springer, Dordrecht, The Netherlands.

Gindro, K., Pezet, R., Viret, O., and Richter, H. 2005. Development of a rapid and highly sensitive direct-PCR assay to detect a single conidium of $\mathrm{Bo}$ trytis cinerea Pers.: Fr in vitro and quiescent forms in planta. Vitis Geilweilerhof 44:139-142.

González-Domínguez, E., Caffi, T., Ciliberti, N., and Rossi, V. 2015. A mechanistic model of Botrytis cinerea on grapevines that includes weather, vine growth stage, and the main infection pathways. PLoS One 10:e0140444.

González-Domínguez, E., Fedele, G., Caffi, T., Delière, L., Sauris, P., Gramaje, D., Ramos-Saez de Ojer, J. L., Díaz-Losada, E., Díez-Navajas, A. M., Bengoa, P., and Rossi, V. 2019. A network meta-analysis provides new insight into fungicide scheduling for the control of Botrytis cinerea in vineyards. Pest Manag. Sci. 75:324-332.
Gusberti, M., Patocchi, A., Gessler, C., and Broggini, G. A. L. 2012. Quantification of Venturia inaequalis growth in Malus $\times$ domestica with quantitative real-time polymerase chain reaction. Plant Dis. 96:17911797.

Hill, G. N., Evans, K. J., Beresford, R. M., and Dambergs, R. G. 2014. Comparison of methods for the quantification of Botrytis bunch rot in white wine grapes. Aust. J. Grape Wine Res. 20:432-441.

Holz, G., Gütschow, M., Coertze, S., and Calitz, F. J. 2003. Occurrence of Botrytis cinerea and subsequent disease expression at different positions on leaves and bunches of grape. Plant Dis. 87:351-358.

Jaspers, M. V., Seyb, A. M., Trought, M. C. T., and Balasubramaniam, R. 2013. Overwintering grapevine debris as an important source of Botrytis cinerea inoculum. Plant Pathol. 62:130-138.

Keller, M., Viret, O., and Cole, F. M. 2003. Botrytis cinerea infection in grape flowers: Defense reaction, latency, and disease expression. Phytopathology 93:316-322.

Lorenz, D., Eichhorn, K., Bleiholder, H., Klose, R., Meier, U., and Weber, E. 1994. Phänologische Entwicklungsstadien der Weinrebe (Vitis vinifera L. ssp. vinifera). Vitic. Enol. Sci. 49:66-70.

McClellan, W. D., and Hewitt, W. B. 1973. Early Botrytis rot of grapes: Time of infection and latency of Botrytis cinerea Pers. in Vitis vinifera L. Phytopathology 63:1151-1157.

Ministero delle politiche agricole alimentari, forestali e del turismo. 2017. Linee guida nazionali di produzione integrata delle colture, 2018. https:// www.reterurale.it/flex/cm/pages/ServeBLOB.php/L/IT/IDPagina/17765

Mullins, M. G., and Rajasekaran, K. 1981. Fruiting cuttings: Revised method for producing test plants of grapevine cultivars. Am. J. Enol. Vitic. 32: $35-40$.

Mundy, D. C., Agnew, R. H., and Wood, P. N. 2012. Grape tendrils as an inoculum source of Botrytis cinerea in vineyards - a review. N. Z. Plant Prot. 65:218-227.

Nair, N., Guilbaud-Oulton, S., Barchia, I., and Emmett, R. 1995. Significance of carry over inoculum, flower infection and latency on the incidence of Botrytis cinerea in berries of grapevines at harvest in New South Wales. Aust. J. Exp. Agric. 35:1177-1180.

Pertot, I., Caffi, T., Rossi, V., Mugnai, L., Hoffmann, C., Grando, M. S., Gary, C., Lafond, D., Duso, C., Thiery, D., Mazzoni, V., and Anfora, G. 2017. A critical review of plant protection tools for reducing pesticide use on grapevine and new perspectives for the implementation of IPM in viticulture. Crop Prot. 97:70-84.

Pezet, R., Viret, O., Perret, C., and Tabacchi, R. 2003. Latency of Botrytis cinerea Pers.: Fr. and biochemical studies during growth and ripening of two grape berry cultivars, respectively susceptible and resistant to grey mould. J. Phytopathol. 151:208-214.

Saito, S., Dunne, K. J., Evans, K. J., Barry, K., Cadle-Davidson, L., and Wilcox, W. F. 2013. Optimisation of techniques for quantification of Botrytis cinerea in grape berries and receptacles by quantitative polymerase chain reaction. Aust. J. Grape Wine Res. 19:68-73.

Sanzani, S. M., Schena, L., De Cicco, V., and Ippolito, A. 2012. Early detection of Botrytis cinerea latent infections as a tool to improve postharvest quality of table grapes. Postharvest Biol. Technol. 68:64-71.

Seyb, A., Gaunt, R., Trought, M., Frampton, C., Balasubramaniam, R., and Jaspers, M. 2000. Relationship between debris within grape bunches and Botrytis infection of berries. Page 451 in: Proceedings of the 1998 New Zealand Plant Protection Conference. New Zealand Plant Protection Society, Auckland, New Zealand.

Suarez, M. B., Walsh, K., Boonham, N., O’Neill, T., Pearson, S., and Barker, I. 2005. Development of real-time PCR (TaqMan $\left.{ }^{\circledR}\right)$ assays for the detection and quantification of Botrytis cinerea in planta. Plant Physiol. Biochem. 43: 890-899.

Valsesia, G., Gobbin, D., Patocchi, A., Vecchione, A., Pertot, I., and Gessler, C. 2005. Development of a high-throughput method for quantification of Plasmopara viticola DNA in grapevine leaves by means of quantitative real-time polymerase chain reaction. Phytopathology 95:672-678.

Viret, O., Keller, M., Jaudzems, V. G., and Cole, F. M. 2004. Botrytis cinerea infection of grape flowers: Light and electron microscopical studies of infection sites. Phytopathology 94:850-857.

Wolf, T. K., Baudoin, A. B. A. M., and Martinez-Ochoa, N. 1997. Effect of floral debris removal from fruit clusters on Botrytis bunch rot of Chardonnay grapes. Vitis 16:27-33. 